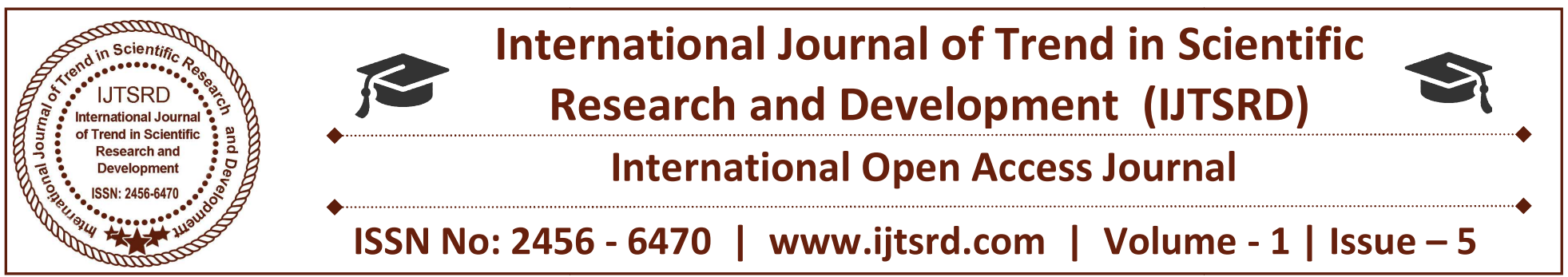

\title{
Fuzzy Logic Based Parameter Adaptation of Interior Search Algorithm
}

\author{
Dr.Prabha Shreeraj Nair \\ Dean Research \\ Tulsiramji Gayakwade Patil College of Engineering and Technology, Nagpur
}

\begin{abstract}
Interior Search Algorithm (IS A) is a novel algorithm recently developed for solving optimization problems. Though, this metaheuristic has proven to be effective for function optimization on widely used benchmark functions, this algorithm may converge slowly or be trapped in local minima for complex functions. In this paper, a new hybrid approach of IS A is presented. The proposed approach includes a fuzzy logic system for improving convergence of the solutions in IS A by dynamic parameter adaptation. To validate the performance of the proposed algorithm, several comparisons are presented on the basis of benchmark functions. Simulation results demonstrated the efficiency of the proposed fuzzy IS A algorithm.
\end{abstract}

Keywords - interior search algorithm; fuzzy logic; parameter adaptation; benchmark functions

\section{INTRODUCTION:}

Optimization techniques provide an important paradigm for solving real-world complex problems. In an engineering environment, optimization process includes searching for a vector that represents an optimal solution relative to others for a specific objective function. Optimization processes can be divided into two main categories: deterministic and heuristic. The former involves rigid movements which are not adaptable paper is the proposed Fuzzy ISA (FISA) with dynamic adaptation of parameters by studying the effect of iteration count on convergence and accuracy of solutions while updating the parameter.
This paper is organized as follows; Section II presents the ISA algorithm, Section III presents the proposed technique, Section IV presents the simulation results and Section $\mathrm{V}$ concludes and presents future research.

\section{INT ERIOR SEARCH ALGORITHM}

The main inspiration for the proposed method ISA is the manner of arrangement of objects in the room in order to improve the overall aesthetics known as interior designing. This method is carried out in accordance with the requirements and limitations set by the client [11].

Keeping in mind the aesthetics, the designer tries to replicate the best features of the room which can be used as an inspiration for optimization. It may be noted that the algorithmic procedure can be mathematically modeled by the following processes:

According to the convexity, continuity and differentiability of the problem. On the other hand, heuristic undertake stochastic movements which are flexible, derivative free and therefore provide optimal solutions with higher efficiency. The main categories include Evolutionary algorithms, Swarm intelligence and Physics based algorithms [1]. Recently, heuristic methods inspired by human behavior have been extensively studied and they provide a convenient mechanism for dealing with different optimization problems. Some of the popular algorithms include Teacher Learning Based Optimization (TLBO) [2], Tabu Search (TS) [3], Imperialistic Competitive 
Algorithm (ICA ) [4], Harmony Search (HS) [5], Mine Blast Algorithm [6], Seeker Optimization Algorithm (SOA) [7], Social-Based Algorithm (SBA) [8] and Group Search Optimizer (GSO) [9].

Advantages of these algorithms range from low number of tuning parameters [2][3], less number of operators than Evolutionary techniques (elitism, mutation etc.) to low dependence on few individual solutions (global best, hierarchical structure etc.) to guide the search like in Swarm intelligence.

The Interior Search Algorithm (ISA) [10] is a human inspired evolutionary computing based algorithm, recently proposed by Gandomi in 2014. The main contribution of this Mirror work.

In this algorithm, solution set is divided into two groups, wherein, the composition group, the composition of solutions is modified to find better solutions and the mirror group, where mirrors are placed between the fittest element and other elements to find better views. This division is controlled by a parameter ' $\alpha$ ' which varies from a range of 0 to 1 . Since, ' $\alpha$ ' is the only parameter of the algorithm, it is important for the distribution between the diversification and intensification search carried out by the algorithm.

\section{A. Composition design}

Composition or interior design follows a structured procedure that includes a coordinated and systematic process. The interior design usually starts from the walls (bounds) towards the center. Therefore, the space becomes more and more limited for designing the composition of the elements (solutions). Since the design space becomes limited at every stage of the procedure, hence only a more beautiful view (fitter solution) will be obtained at each stage due to the limitation s imposed by the client.

\section{B. Mirror Work}

In mirror work, different mirrors are used to provide different views in order to create a more decorative room.

Hence, mirrors are placed next to beautiful elements (fitter will work as a local search or intensification otherwise as a solutions) to emphasize their effectiveness (fitness). This leads global search or diversification which can be observed from to better search around the fittest element(s) as placement of mirrorsnear global best(s) can lead to better views (fitter As shown in this section, ISA possesses a single parameter, solutions). This can be modeled by placing a mirror randomly $\alpha$ which makes the algorithm highly suitable and easily between each element and the global best (fittest solution).

Therefore, the position of a mirror for th element at th iteration can be formulated as,

$$
,=3 \times-1+(1-3) \times
$$

where 3 is a random number between 0 and 1 . Since the virtual image location depends on location of the mirror, it can be formulated as

$$
\begin{array}{lll}
=2 \times & - & -1
\end{array}
$$

Furthermore, the position of the global best is changed using a random walk as local search. This can be formulated as ,

$$
=-1+\times
$$

where is a scale factor depending on search space and is a normally distributed vector of random numbers. The updated elements (global or otherwise) and images (virtual elements) are retained only if the fitness is improved compared to earlier position.

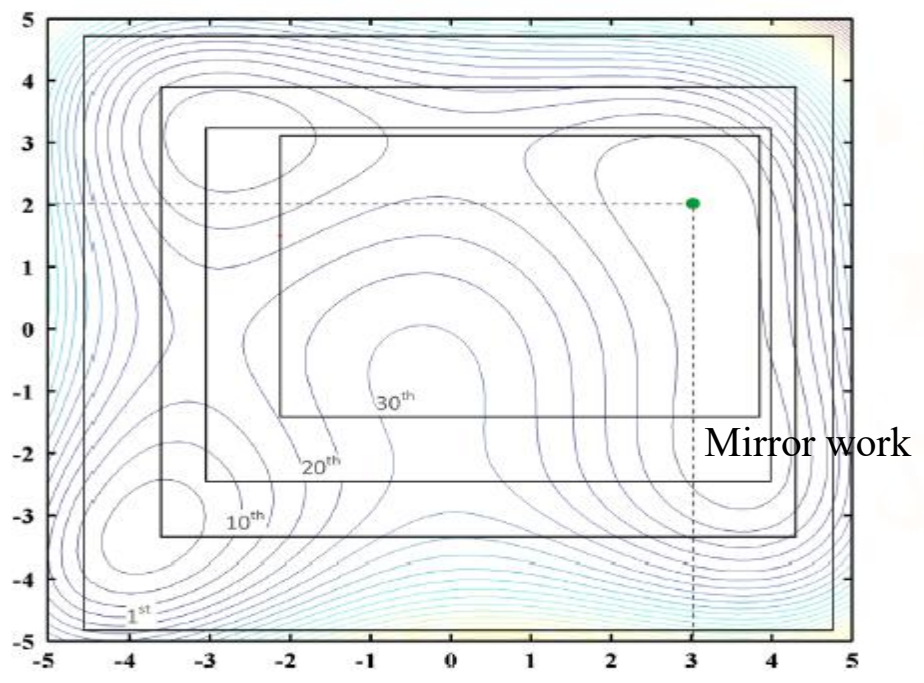

Fig.1 Composition stages for 2D Himmelblau function 


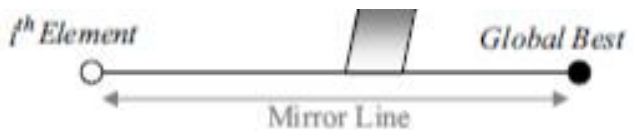

(a)

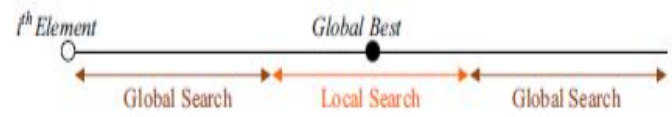

(b)

Fig.2 Mirror work search part of algorithm

For an image placed near the global best, the mirror search will work as a local search or intensification otherwise as a global search or diversification

Fig. 2. The pseudo code of ISA is shown in Fig 3.

\section{DYNAMIC ADAPTATION OF PARAMETER ALPHA}

Fuzzy logic proposed by Prof. Lotfi Zadeh in 1965 is based on fuzzy set theory. Fuzzy logic helps in modelling knowledge through the use of if-then fuzzy rules.

Initialize the solutions randomly $x_{i}(\mathrm{i}=1,2, \mathrm{n})$
Initialize parameter $\alpha$
while (j< Max number of iterations)
for $\mathrm{i}=1$ to $\mathrm{n}$
Calculate fitness and find best $x_{g b}^{j}$
if $x_{g b}^{j}$, Update $x_{g b}^{j}$ using Eq (4)
else if rand (0,1) $\leq \alpha$
$\quad$ Update $x_{i}^{j}$ usingEq (2) \& Eq (3)
else
$\quad$ Update $x_{i}^{j}$ using Eq (1)
Calculate fitness of $x_{i}^{j}$, and if better than
$x_{i}^{j-1}$, replace $x_{i}^{j-1}$ with $x_{i}^{j}$
endfor
Update $\alpha$
$\mathrm{j}=\mathrm{j}+1$
endwhile

Through the use of linguistic information, fuzzy set theory provides a convenient method for numerical computations by the use of linguistic labels given by membership functions [12]. Therefore, the proposed technique makes use of a fuzzy inference system (FIS) to update the value of $\alpha$. In order to properly assess the runs of an algorithm, the iterations and diversity of population need to be taken into consideration. The main metrics about ISA that are used are iteration and diversity. Therefore, the aforementioned metrics are used as input variables for the fuzzy logic system to adaptively change $\alpha$.

For this purpose, iteration is defined using a representation of percentage of populatiowhichichncke observe be formulated as ,

where is the total number of iterations set for ISA and is the number of elapsed iterations. This metric is used since it allows us to control the behavior of ISA to perform a global search in earlier iterations followed by local search in later iterations. The range of input variable Iteration is from 0 to 1 since it can only be $0-100 \%$ as observed from Eq. (4). Therefore, while starting the algorithm, the iteration will be close to 0 and can be considered "low", and when completed it will be close to 1 and can be considered "high". The granularity of this metric onto three triangular functions can be appreciated from Fig. 4.

The next metric to be considered is Diversity which is a measure of dispersion of the solutions. This can be formulated as,

$$
\left.(())={ }_{\sum_{F^{\perp}} \sum_{=1}^{1}} \sum^{-}(-\overline{(})\right)^{2}
$$

Where is the current iteration, is the number of solutions, is the total number of dimensions, is the size of the population, is the dimension of the solution and the dimension of the current best solution of the population. This metric is considered since it can control the adaptability of the algorithm to a specific problem, with respect to the diversity of the solutions being obtained. It can lead to emphasis of exploitation when solutions are closer together due to diversity being "high" and exploration when solutions are separated from each other due to diversity being "low". As can be seen from Eq. (5), the range of diversity will range from a value of 0 for "low" to a value of 1 for "high". 
The type and number of membership functions are based on experimental work carried out in [13]. It may be noted that the different type of membership functions do not show much difference in results.

The output variable parameter $\alpha$ is reported to have a value between 0 and 1 . This behaviour was granulated using five membership functions which resulted in five levels of "Low", "MediumLow", "Medium", "MediumHigh" and "High", as shown in Fig. 6. The ranges that are mentioned for ISA that is available in the existing literature,

$[0.1,0.3]$

$[0.1,0.9]$

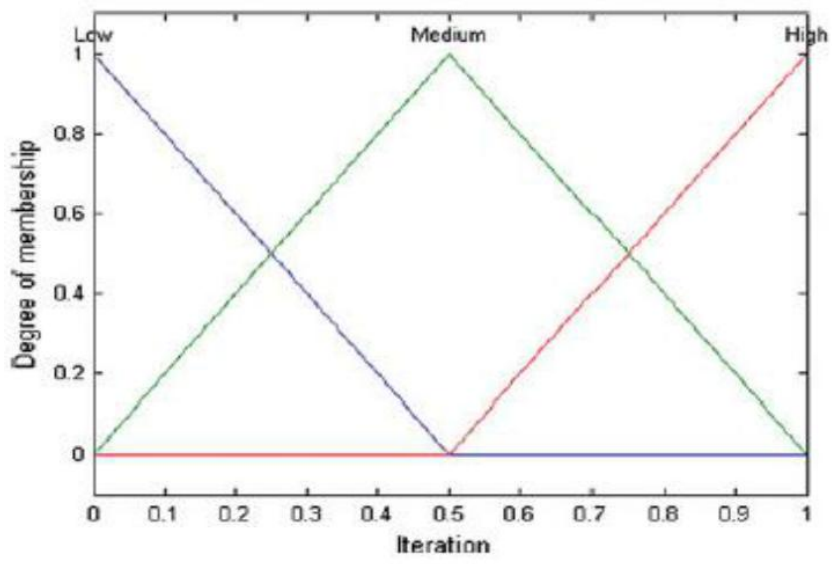

Fig.4 Variable Iteration as input to FIS

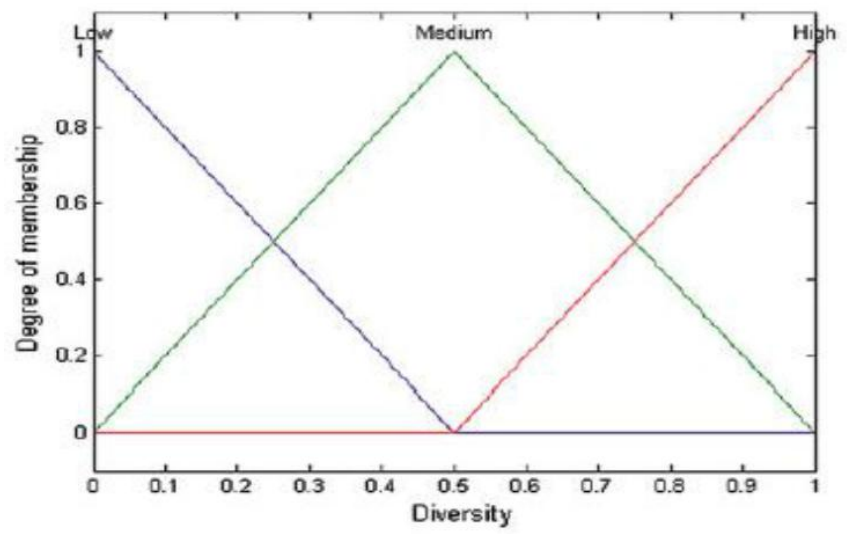

Fig.5 Variable Diversity as input to FIS

In the proposed technique, $\alpha$ is linearly increasing in earlier stages of the optimization process, since a lower $\alpha$ will provide more exploration compared to a higher $\alpha$ at later stages of iterations which will lead to a higher exploitation. This type of division between diversification and intensification is essentially critical for any algorithm's performance. The range
$[0.1,0.3]$ has been claimed in [10] to be superior to other ranges, hence this setting will be followed for conventional ISA. The range for FISA has been set as $[0.1,0.9]$ for careful adaptation of parameter $\alpha$ according to the contours of the op timization problem.

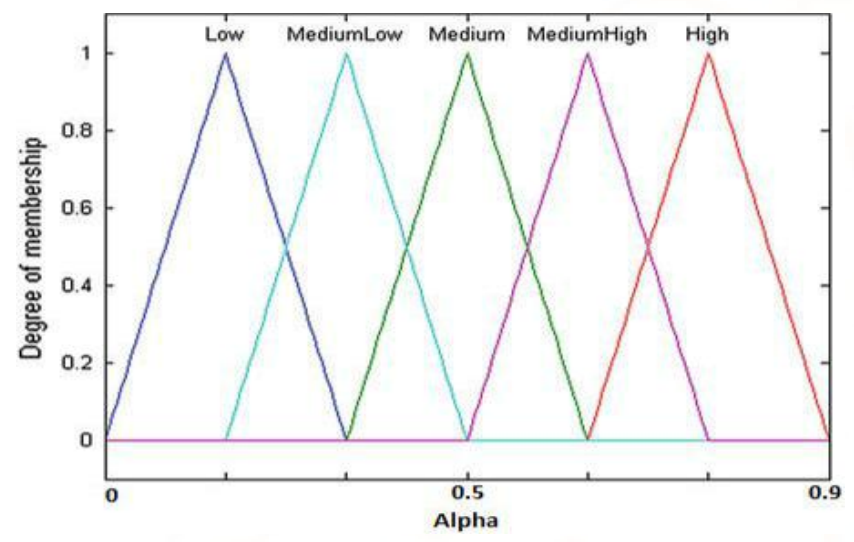

Fig.6 Variable Alpha as output to FIS

\section{Rule set used in FIS}

1. If (Iteration is Low) and (Diversity is Low), then alpha is Low.

2. If (Iteration is Low) and (Diversity is Medium), then alpha is MediumLow.

3. If (Iteration is Low) and (Diversity is High), then alpha is Medium.

4. If (Iteration is Medium) and (Diversity is Low), then alpha is MediumLow.

5. If (Iteration is Medium) and (Diversity is Medium), then alpha is Medium.

6. If (Iteration is Medium) and (Diversity is High), then alpha is MediumHigh.

7. If (Iteration is High) and (Diversity is Low), then alpha is Medium.

8. If (Iteration is High) and (Diversity is Medium), then alpha is MediumHigh.

9. If (Iteration is High) and (Diversity is High), then alpha is High. 
It may be noted that the five triangular membership functions in the output is due to the fact that with two inputs along with their three triangular membership functions results in nine rules and operated on by "and" operator. The rule set considered in this proposed method is shown in Fig. 7

Furthermore, these five membership functions along with the rule set represent the ideal behavior that needs to be embedded in the ISA search procedure. This can be seen from the fact that ISA should have high exploration in initial iterations, high exploitation in final iterations, high exploration with low diversity and high exploitation with high diversity of solutions. Therefore, ISA performs a global search initially when the iterations are low followed by local search in best area found by algorithm. The effect of diversity allows ISA to escape local minima since if all solutions are in a very small area and not in final iterations, then these solutions will be forced to separate by encouraging exploration among solutions due to fuzzy logic.

It may be noted that this particular integration of FIS is similar to the tuning of parameters as done by an experienced user of any algorithm. Hence, FIS can be effectively used to model complex landscapes of optimization problems.

The final fuzzy system is shown in Fig. 8 and it can be seen that the FIS used is Mamdani type along with the required rule set.

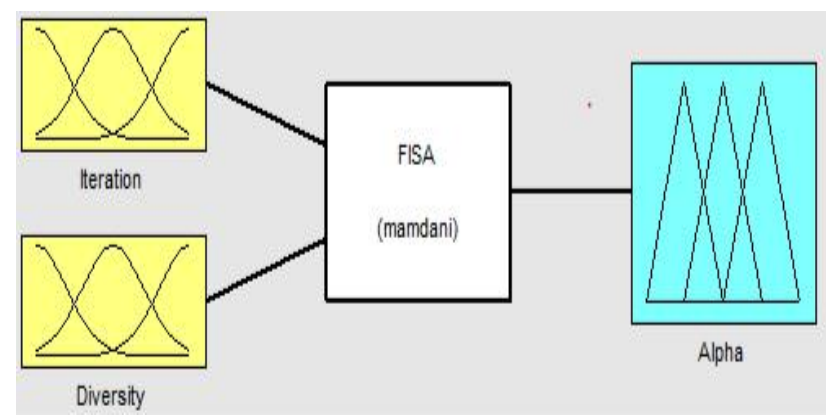

Fig.8 Fuzzy Inference System for ISA

\section{SIMULAT IONS AND EXPERIMENTS}

This section presents the used benchmark functions followed by the obtained results.

\section{A. Benchmark functions}

The benchmark functions considered in this paper are explained in [1] [10] [14] [15] which the reader can refer to for a brief introduction. A total of ten benchmark functions were used for the simulations consisting of six unimodal and four multimodal functions.

Table 1 presents all the used benchmark functions along with ranges considered and Fig 9 shows the plots of these functions in three dimensions.

In all the performed experiments, the parameters used for ISA, $\alpha$ is $[0.1,0.3]$ as in [10] and for FISA, $\alpha$ is dynamically adapted using the fuzzy inference system discussed in the previous sections. The population settings for both were kept as 50 for unimodal functions and 100 for multimodal functions. The total number of iterations is set as 1000 for unimodal functions and 2000 for multimodal functions .

\section{TABLE I. BENCHMARK FUNCTIONS}

TABLE I. BENCHMARK FunCtions

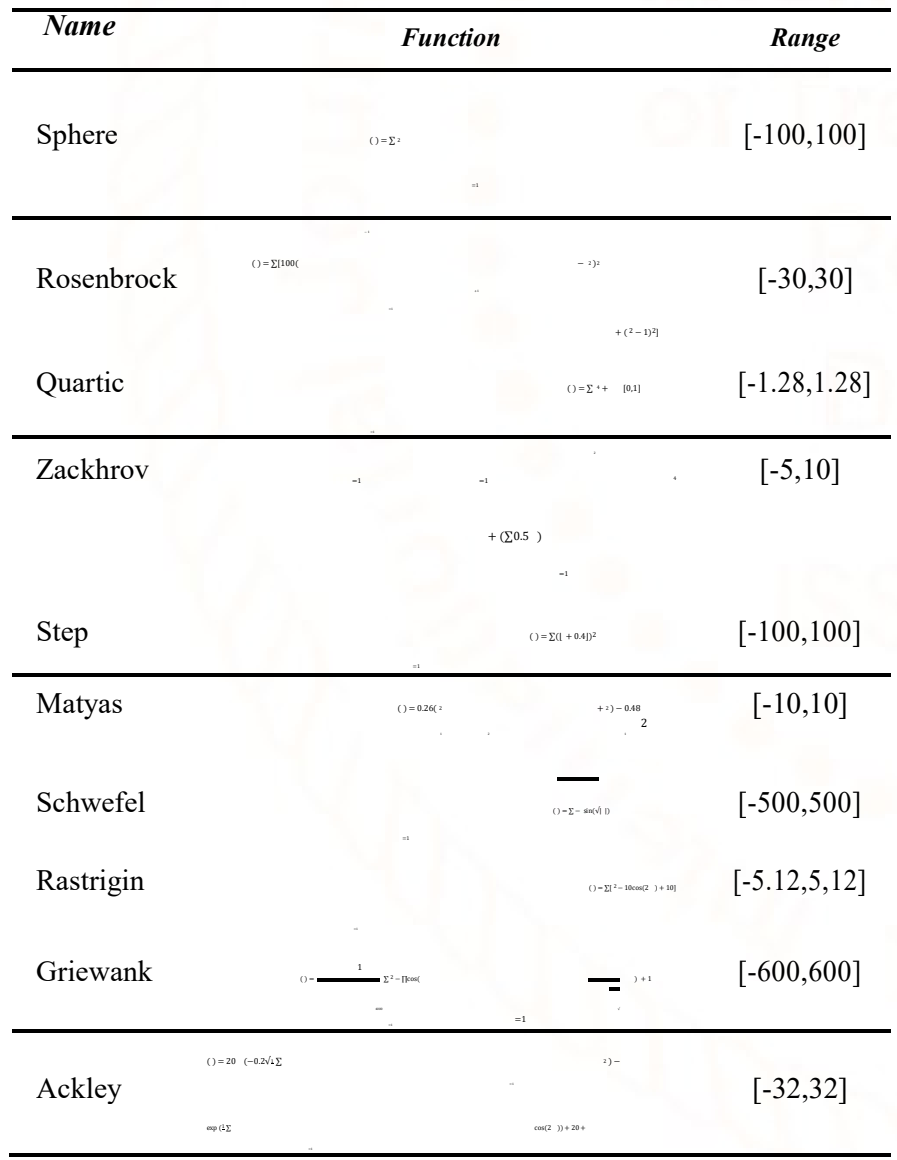

Since ISA is a stochastic process, a single run is not sufficient to verify the algorithm's robustness and accuracy; therefore 100 trial runs are conducted and the results are presented in the following sections. 
International Journal of Trend in Scientific Research and Development (IJTSRD) ISSN: 2456-6470

\section{B. Simulation Results}

The simulation results are presented in this section wherein the best, worst and the mean fitness of 100 trials is presented along with the standard deviation obtained by each algorithm.

TABLE II. RESULTS OF FISA

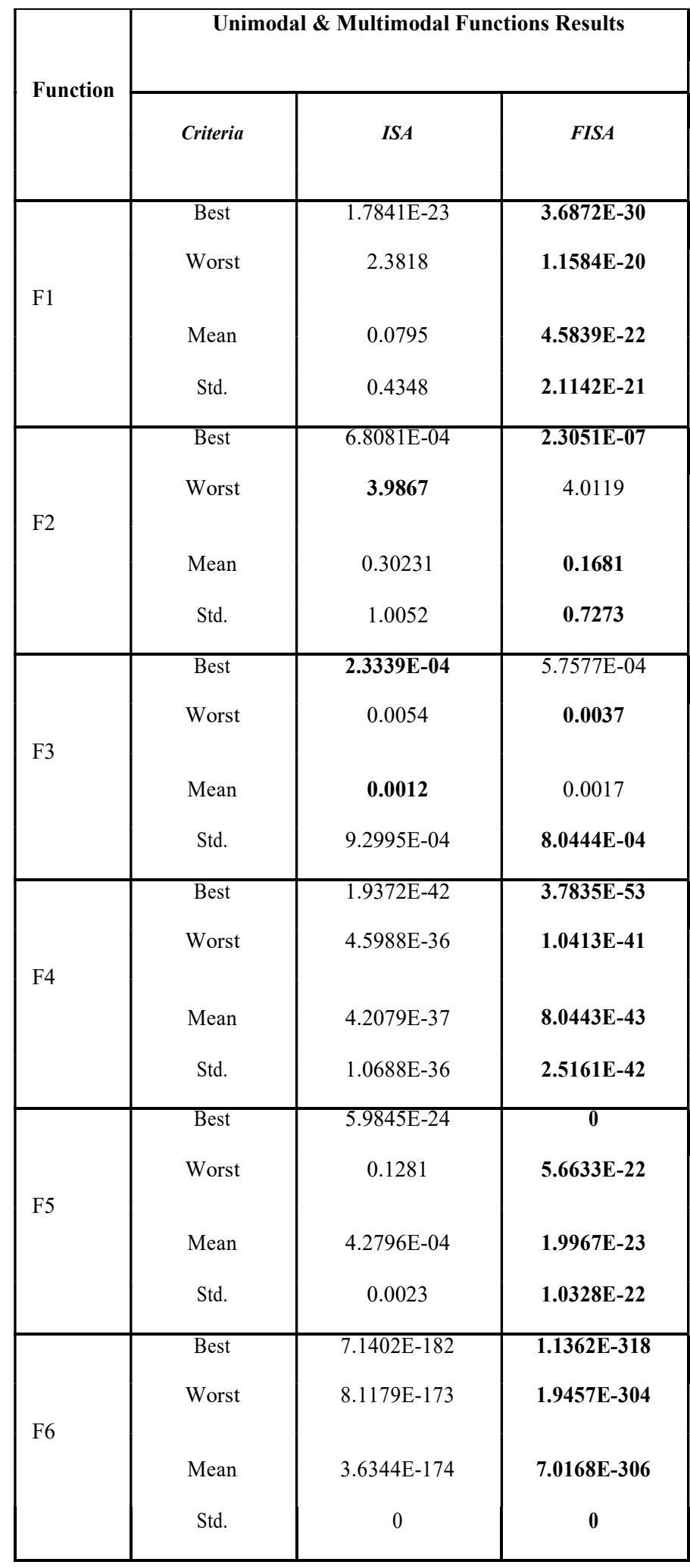

\begin{tabular}{|c|c|c|c|}
\hline F7 & $\begin{array}{l}\text { Best } \\
\text { Worst } \\
\text { Mean } \\
\text { Std. }\end{array}$ & $\begin{array}{l}-7.1876 \mathrm{E}+03 \\
-4.3118 \mathrm{E}+03 \\
-5.8133 \mathrm{E}+03 \\
6.9845 \mathrm{E}+03\end{array}$ & $\begin{array}{r}-7.6666 \mathrm{E}+03 \\
-4.6486 \mathrm{E}+03 \\
-5.9116 \mathrm{E}+03 \\
6.8444 \mathrm{E}+03\end{array}$ \\
\hline F8 & $\begin{array}{c}\text { Best } \\
\text { Worst } \\
\text { Mean } \\
\text { Std. }\end{array}$ & $\begin{array}{l}4.9747 \\
27.8588\end{array}$ & $\begin{array}{r}3.9798 \\
26.8638\end{array}$ \\
\hline F9 & $\begin{array}{c}\text { Best } \\
\text { Worst } \\
\text { Mean } \\
\text { Std. }\end{array}$ & $\begin{array}{l}0.0344 \\
0.4999\end{array}$ & \begin{tabular}{|l|}
0.0295 \\
0.3887 \\
0.1428 \\
0.0815 \\
\end{tabular} \\
\hline F10 & $\begin{array}{c}\text { Best } \\
\text { Worst } \\
\text { Mean } \\
\text { Std. }\end{array}$ & $\begin{array}{l}4.4408 \mathrm{E}-15 \\
4.4408 \mathrm{E}-15\end{array}$ & $\begin{array}{l}8.8817 \mathrm{E}-16 \\
4.4408 \mathrm{E}-15\end{array}$ \\
\hline
\end{tabular}

As can be seen from above table, FISA is able to efficiently converge in the case of unimodal functions. FISA achieves a much lower best, mean, worst and standard deviation, especially for F1, F2, F4 and F5. It may be noted that FISA is able to converge to an optimum of 0 in the case of F5 which highlights the high exploration as well as exploitation undertaken by FISA relative to ISA. In the case of multimodal functions, FISA always performs better than ISA by a small margin. This is due to the complex nature of the search space of multimodal functions. A notable point may be the performance of FISA on the benchmark function F10 wherein FISA is able to escape local minima to converge to a lower best and overall mean compared to ISA's performance on that complex benchmark function. 


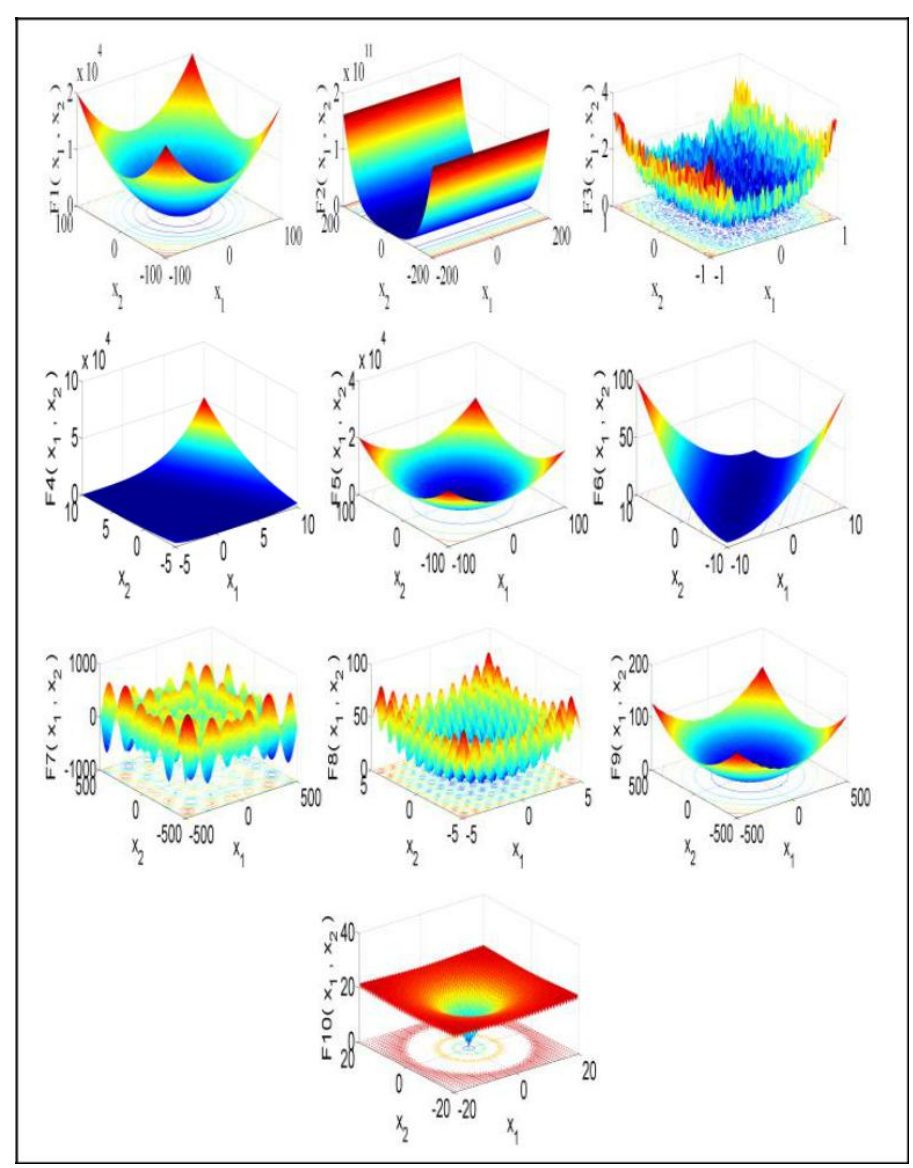

Fig. 9 Plots of benchmark functions

\section{CONCLUSION}

This paper presents an application of fuzzy logic to improve the performance of metaheuristic ISA by dynamically adapting the parameter of ISA, $\alpha$. The simulation results demonstrate the efficiency of FISA in dealing with complex benchmark functions and showcase the overall superiority of proposed technique of FISA over ISA. It may be noted that addition of FIS to ISA does not incur an increase in $\mathrm{s}$ pace and time complexity. Also, the use of FIS to adapt $\alpha$ does not lead to an increase in the number of function evaluations as can be seen due to addition of operators such as mutation, crossover, oppositionbased learning etc. For future research work, the proposed technique FISA can be applied to real world applications like spring design, cantilever beam optimization etc. Moreover, fuzzy type 2 can also be explored in adapting the parameter $\alpha$.

\section{REFERENCES}

[1] Mirjalili, Seyedali, and Andrew Lewis. "T he whale optimization algorithm." Advances in Engineering Software 95 (2016): 51-67.

[2] Rao, Ravipudi V., Vimal J. Savsani, and D. P. Vakharia. "T eaching- learning-based optimization: a novel method for constrained mechanical design optimization problems." Com puter-Aided Design 43.3 (2011): 303-315.

[3] Glover, Fred. "T abu search-part I." ORSA Journal on computing 1.3 (1989): 190-206.

[4] Atashpaz-Gargari, Esmaeil, and Caro Lucas. "Imperialist competitive algorithm: an algorithm for optimization inspired by imperialistic competition." Evolutionary computation, 2007. CEC 2007. IEEE Congress on. IEEE, 2007.

[5] Geem, Zong Woo, Joong Hoon Kim, and G. V. Loganathan. "A new heuristic optimization algorithm: harmony search." Simulation 76.2 (2001): 60-68.

[6] Sadollah, Ali, Ardeshir Bahreininejad, Hadi Eskandar, and Mohd Hamdi. "Mine blast algorithm: A new population based algorithm for solving constrained engineering optimization problems."

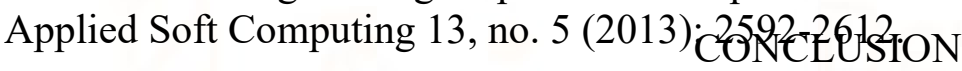

[7] Dai, Chaohua, Yunfang Zhu, and Weirong Chen. "Seeker optimization algorithm." International Conference on Computational and Information Science. Springer Berlin Heidelberg, 2006.

[8] Ramezani, Fatemeh, and Shahriar Lotfi. "Social-based algorithm (SBA)." Applied Soft Computing 13.5 (2013): 2837-2856.

[9] He, Shan, Q. Henry Wu, and J. R. Saunders. "Group search optimizer: an optimization algorithm inspired by animal searching behavior." IEEE transactions on evolutionary computation 13.5 (2009): 973-990.

[10] Gandomi, Amir H. "Interior search algorithm (ISA): a novel approach for global optimization." ISA transactions 53.4 (2014): 1168-1183..

[11] NCIDQ2017.

Retrieved Feb. 1,2017.

〈http://www.ncidq.org〉

[12] Abdelbar, Ashraf M., Suzan Abdelshahid, and Donald C. Wunsch. "Fuzzy PSO: a generalization of particle swarm optimization." Neural Networks, 2005. 
IJCNN'05. Proceedings. 2005 IEEE International Joint Conference on. Vol. 2. IEEE, 2005.

[13] Olivas, Frumen, Fevrier Valdez, and Oscar Castillo. "A Comparative Study of Membership Functions for an Interval T ype-2 Fuzzy System Used for Dynamic Parameter Adaptation in Particle Swarm Optimization." Recent Developments and New Direction in Soft-Computing Foundations and Applications. Springer International Publishing, 2016. 373-385.
[14] J. Digalakis and K. Margaritis, “On benchmarking functions for genetic algorithms", Int J Comput Math, vol. 77, 2001, pp. 481 -506.

[15] Molga, Marcin, and Czesław Smutnicki. "Test functions for optimization needs." Test functions for optimization needs (2005). 